\title{
Resonance behavior of internal conversion coefficients at low $\gamma$-ray energy
}

\author{
M. B. Trzhaskovskaya, ${ }^{1}$ T. Kibédi, ${ }^{2}$ and V. K. Nikulin ${ }^{3}$ \\ ${ }^{1}$ Petersburg Nuclear Physics Institute, RU-188300 Gatchina, Russia \\ ${ }^{2}$ Department of Nuclear Physics, Research School of Physics and Engineering, The Australian National University, \\ Canberra, ACT 0200, Australia \\ ${ }^{3}$ Ioffe Physical Technical Institute, 26 Polytekhnicheskaya, RU-194021 St. Petersburg, Russia
}

(Received 3 December 2009; published 26 February 2010)

\begin{abstract}
A resonance-like structure of internal conversion coefficients (ICCs) at low $\gamma$-ray energy $(\lesssim 100 \mathrm{keV})$ is studied. Our calculations revealed new, previously unknown resonance minima in the energy dependence of ICCs for the $n s$ shells at E2-E5 transitions. The resonances are the most defined for ICCs in light and medium elements with $Z \lesssim 50$. It is shown that ICCs may have up to four resonances for outer shells while it has been assumed so far that only one resonance exists. Well-pronounced resonances in ICCs at $E 1$ transition were discovered for the $n s$ shells with $n \geqslant 2$ as well as for the $n p$ shells with $n \geqslant 3$ and the $n d$ shells with $n \geqslant 4$ of all elements up to superheavy ones. Simple expressions for approximate values of the $E 1$ resonance energy were obtained which are of importance for determination of the resonance energy range where the interpolation of ICCs taken from tables or databases may give significant errors. The occurrence of resonances in ICCs is explained by vanishing conversion matrix elements under changes of sign. The peculiarities of the behavior of the matrix elements and electron wave functions at the resonance energy are considered. Available experimental ICCs for electric transitions with energies near the expected position of resonances satisfactory agree with our calculations.
\end{abstract}

DOI: 10.1103/PhysRevC.81.024326

PACS number(s): $23.20 . \mathrm{Nx}$

\section{INTRODUCTION}

Internal conversion coefficients (ICCs) are commonly used in solving many problems of nuclear physics. Through comparison of experimental ICCs with corresponding theoretical values, multipolarities and mixing ratios of nuclear transitions are determined. Precise values of ICCs are also required for the development of nuclear level schemes as well as decay schemes, in Mössbauer spectroscopy [1], in the decay heat calculations of spent nuclear reactor fuel cells [2], and in other nuclear physics research. With a further increase of resolving power to the magnitude of the order of natural line widths of valence electrons $(\lesssim 1 \mathrm{eV})$, the conversion electron spectroscopy provides a method for study of the electronic structure of condensed matter [3-5]. So the low-energy nuclear transitions are of special interest currently [6-8]. Several tens of transitions with the energy $E_{\gamma} \lesssim 5 \mathrm{keV}$ are investigated experimentally and theoretically up to the uniquely low-lying isomer ${ }^{229} \mathrm{Th}^{m}$ with energy of several eV [9,10]. All studies just mentioned require a knowledge of accurate values of theoretical ICCs.

There are a number of widely used ICC calculations. The recent tables [11] are recognized to be the most reliable ones. The calculation is based on the Dirac-Fock (DF) method where the exchange interaction is considered exactly between atomic electrons as well as between bound and continuum electrons. Data from Ref. [11] revealed the excellent average agreement with available the most accurate experimental ICC values [12]. However, some of individual ICCs disagreed considerably with theoretical DF values, initiating new ICC measurements with better than $1 \%$ precision [13-16] to improve the experimental values and to test the theoretical model underlying the ICC calculations. Experimental ICC values obtained are in excellent agreement with DF calculations having regard to a vacancy in the atomic subshell where conversion occurs.

To make the new theoretical ICCs accessible for a very broad user community, the ICC database BrIcc has been developed [17,18] which is now adopted by International Network of Nuclear Structure and Decay Data (NSDD). As distinct from that in Ref. [11], a calculation in Ref. [17] has been performed with regard to the vacancy. In the new tabulations, the $\gamma$-ray energy range under consideration has been extended. ICC values for a specific transition can be obtained by interpolation within the BrIcc database.

It is common knowledge that ICC for a specific atomic shell is usually a monotonic function of the $\gamma$-ray energy $E_{\gamma}$, the multipolarity $L$, and the nuclear charge $Z$ [19]. However, at low energies, the monotony may break down. Of particular importance is the nonmonotonic behavior of ICCs as a function of $E_{\gamma}$. All ICC tables are based on an energy mesh and ICCs for a specific energy are obtained by interpolation. In regions where the ICC is a nonmonotonic function of $E_{\gamma}$ (so-called resonance regions), one can obtain erroneous ICC values as a result of interpolation [20,21]. It should be noted that, in addition, total ICCs may be nonmonotonically behaved showing "toothed discontinuities" when $E_{\gamma}$ passes through thresholds of relevant subshells [11,22,23]. However, we are concerned here with a resonance structure of the subshell ICCs.

Church and Monahan [24] were the first to note that the ICCs in the $L_{1}$ and $M_{1}$ shells for electric transitions are nonmonotonic as a function of $E_{\gamma}$. More recently, the problem was further discussed in Refs. [12,20-22]. It was noted in Refs. $[12,22,24]$ that the nonmonotonic behavior of ICCs is a result of the matrix element cancellation. However, it has been assumed so far that one minimum in the $E_{\gamma}$ dependence of ICC exists only in the case of the $n s$ shells with the principal 
quantum number $n \geqslant 2$ and for $L \geqslant 2$ electric transitions in $Z \lesssim 50$ elements (see, for example, Ref. [12]). The case of the $E 1$ resonance was found by Dragoun et al. in ICC for the $5 d_{3 / 2}$ subshell of $\mathrm{Pa}(Z=91)$ [25].

In the present article, we report that at low energies, ICCs in the $n s$ shells for $E 2-E 5$ transitions may have more than one resonance. The preliminary results have been presented in Ref. [17]. It has been revealed that this type of resonance occurs not only in light to medium elements but in heavy (up to $Z=100$ ) systems as well. We have also discovered that resonance behavior at $E 1$ transition can be present for the $n s$ shells with $n \geqslant 2$, for the $n p_{1 / 2}$ and $n p_{3 / 2}$ shells with $n \geqslant 3$ as well as for the $n d_{3 / 2}$ and $n d_{5 / 2}$ shells with $n \geqslant 4$ for elements from light to superheavy ones. Consequently, the present article is the first exhausting study of the problem covering a wide range of elements and shells.

In Sec. II, expressions for the ICC calculation are described. In Secs. III and IV, we review all ICC resonances obtained and discuss in detail the peculiarities and reasons of their occurrence. Section V is devoted to a comparison of theoretical values with available experimental ICC ratios of the $L$ subshells $L_{1} / L_{2}$ for electric nuclear transitions with energies near the expected position of resonances to provide support for the existence of the resonances.

\section{METHOD OF CALCULATION}

The relativistic expression for ICC in the $i$ th atomic subshell, derived in the framework of the first nonvanishing order of perturbation theory and one-electron approximation for a free neutral atom, can be written as [11]:

$$
\alpha_{i}^{\tau L}=\sum_{\kappa_{f}}\left|M_{i}^{\tau L}\left(\kappa_{f}\right)\right|^{2} .
$$

The partial conversion matrix element is $M_{i}^{\tau L}\left(\kappa_{f}\right)=$ $B_{i}^{\tau L}\left(\kappa_{f}\right) R_{i}^{\tau L}\left(\kappa_{f}\right)$, where $B_{i}^{\tau L}\left(\kappa_{f}\right)$ is the angular part, $R_{i}^{\tau L}\left(\kappa_{f}\right)$ is the radial part, and $\tau L$ is the nuclear transition multipolarity of electric type $\tau=E$ or magnetic one $\tau=M$. Use is made of relativistic quantum numbers $\kappa=(\ell-j)(2 j+1)$, where $\ell$ is the electron orbital momentum, and $j$ is the total electron momentum. Indices $i$ and $f$ refer to the initial (bound) and final (continuum) states of the electron, respectively.

The summation in Eq. (1) extends over all final states allowed by the selection rules

$$
\begin{gathered}
\left|L-j_{i}\right| \leqslant j_{f} \leqslant L+j_{i}, \\
\ell_{i}+\ell_{f}+L \text { is }\left\{\begin{array}{l}
\text { even for EL transitions, } \\
\text { odd for ML transitions. }
\end{array}\right.
\end{gathered}
$$

For electric transitions, $B_{i}^{\tau L}\left(\kappa_{f}\right)$ and $R_{i}^{\tau L}\left(\kappa_{f}\right)$ are given by

$$
\begin{aligned}
B_{i}^{E L}\left(\kappa_{f}\right)= & (-1)^{j_{f}+\frac{1}{2}+L} C_{\ell_{i} 0 \ell_{f} 0}^{L 0} W\left(\ell_{i} j_{i} \ell_{f} j_{f} ; \frac{1}{2} L\right) \\
& \times\left[\pi k \alpha \frac{\left(2 j_{i}+1\right)\left(2 \ell_{i}+1\right)\left(2 j_{f}+1\right)\left(2 \ell_{f}+1\right)}{L(L+1)(2 L+1)}\right]^{\frac{1}{2}}
\end{aligned}
$$

and

$$
\begin{aligned}
R_{i}^{E L}\left(\kappa_{f}\right)= & \left(\kappa_{i}-\kappa_{f}\right)\left(R_{1, \Lambda=L-1}+R_{2, \Lambda=L-1}\right) \\
& +L\left(R_{2, \Lambda=L-1}-R_{1, \Lambda=L-1}+R_{3, \Lambda=L}\right) .
\end{aligned}
$$

In Eq. (3), $k$ equals $E_{\gamma}$ in unit of $m_{0} c^{2}, \alpha$ is the fine structure constant, $C_{\ell_{i} 0 \ell_{f} 0}^{L 0}$ is the Clebsch-Gordan coefficient, and $W\left(\ell_{i} j_{i} \ell_{f} j_{f} ; \frac{1}{2} L\right)$ is the Racah coefficient.

For magnetic transitions, the corresponding expressions are

$$
\begin{aligned}
B_{i}^{M L}\left(\kappa_{f}\right)= & (-1)^{j_{f}+\frac{1}{2}+L} C_{\ell_{i} 0 \bar{\ell}_{f} 0}^{L 0} W\left(\ell_{i} j_{i} \bar{\ell}_{f} j_{f} ; \frac{1}{2} L\right) \\
& \times\left[\pi k \alpha \frac{\left(2 j_{i}+1\right)\left(2 \ell_{i}+1\right)\left(2 j_{f}+1\right)\left(2 \bar{\ell}_{f}+1\right)}{L(L+1)(2 L+1)}\right]^{\frac{1}{2}},
\end{aligned}
$$

where $\bar{\ell}_{f}=2 j_{f}-\ell_{f}$ and

$$
R_{i}^{M L}\left(\kappa_{f}\right)=\left(\kappa_{i}+\kappa_{f}\right)\left(R_{1, \Lambda=L}+R_{2, \Lambda=L}\right) .
$$

Radial integrals in Eqs. (4) and (6) are written as follows

$$
\begin{aligned}
R_{1, \Lambda} & =\int_{0}^{\infty} G_{i} F_{f}\left(E_{k}\right) X_{\Lambda}(k r) d r, \\
R_{2, \Lambda} & =\int_{0}^{\infty} F_{i} G_{f}\left(E_{k}\right) X_{\Lambda}(k r) d r, \\
R_{3, \Lambda} & =\int_{0}^{\infty}\left[G_{i} G_{f}\left(E_{k}\right)+F_{i} F_{f}\left(E_{k}\right)\right] X_{\Lambda}(k r) d r .
\end{aligned}
$$

Functions $G(r)$ and $F(r)$ are given by $G(r)=r g(r)$ and $F(r)=r f(r)$, where $g(r)$ and $f(r)$ are the large and small components of the radial electron wave function, respectively. In our calculations, $G$ and $F$ are solutions of the DF equations. Wave functions for the bound state $G_{i}$ and $F_{i}$ are calculated in the DF field of a neutral atom while continuum wave functions $G_{f}\left(E_{k}\right)$ and $F_{f}\left(E_{k}\right)$ are determined in the DF field of the ion with a vacancy in the shell from which the conversion electron is emitted. In calculating continuum wave functions, the conversion electron energy $E_{k}$ is determined from the energy conservation relationship:

$$
E_{k}=k-\varepsilon_{i},
$$

where $\varepsilon_{i}$ is the binding energy for the $i$ th atomic subshell. We use the experimental values of $\varepsilon_{i}$. As in the majority of ICC calculations, the recoil energy and the work function are ignored in Eq. (10). These small contributions to $E_{k}$ have no effect on the present study.

The radial part of the transition potential $X_{\Lambda}(k r)$ in the surface current model is written as follows [26]

$$
X_{\Lambda}(k r)=\left\{\begin{array}{lll}
j_{\Lambda}(k r) \frac{h_{\Lambda}\left(k R_{0}\right)}{j_{\Lambda}\left(k R_{0}\right)} & \text { for } \quad r \leqslant R_{0}, \\
h_{\Lambda}(k r) & \text { for } \quad r>R_{0},
\end{array}\right.
$$

where $j_{\Lambda}(x)$ and $h_{\Lambda}(x)$ are the spherical Bessel and Hankel functions, respectively. Inside the nucleus, the potential of a homogeneously charged sphere is assumed where $R_{0}=$ $1.2 A^{1 / 3} \mathrm{fm}$ is the radius and $A$ is the mass number. Eqs. (1)-(11) make use of relativistic units where the electron Compton wavelength $\hbar / m_{0} c$ serves as unit of length and the electron rest energy $m_{0} c^{2}$ as unit of energy. 


\section{NEW ICC RESONANCES}

For a specific multipolarity and a nuclear charge, ICCs usually increase with decreasing energy $E_{\gamma}$ (see Graph I in Ref. [11]). In the appreciable energy region, the $E_{\gamma}$ dependence of ICC $\alpha_{i}^{\tau L}\left(E_{\gamma}\right)$ is close to linear one if the logarithmic scale is used. In spite of the monotonic behavior of the majority of the ICCs as a function of $E_{\gamma}$, it has been noted [24] that there is a minimum in $\alpha_{i}^{E L}\left(E_{\gamma}\right)$ for electric transitions with $L \geqslant 2$ for the $L_{1}$ and $M_{1}$ shells of light and medium elements. This minimum later was called as the "ICC resonance structure" [22], which occurs at the energy $E_{\gamma}^{(\text {res })}$ determined by the following expression for the $L_{1}$ shell [24]:

$$
E_{\gamma}^{(\mathrm{res})}(\mathrm{keV}) \approx \frac{Z^{2}(L-1)}{200} .
$$

We have found [17] that there exist several minima (resonances) in $\alpha_{i}^{E L}\left(E_{\gamma}\right)$ for the $n s$ shells with $n \geqslant 2$, the resonance energy of Eq. (12) being the highest one. In Fig. 1, ICCs for $E 1-E 5$ transitions and the $K, L_{1}, M_{1}$, and $N_{1}$ shells of calcium $(Z=20)$ are presented. The typical $E_{\gamma}$ dependence of ICC for the $K$ shell is demonstrated in Fig. 1(a). The curves $\alpha_{K}^{E L}\left(E_{\gamma}\right)$ fall sharply near the threshold for all transitions except $E 1$. This sharp up-bend of ICC was called "threshold non-regularity" in Ref. [17]. For light elements, the threshold nonregularities occur in ICCs only for electric transitions; however, for more heavy elements, ICC for magnetic transitions also behave in the same way. According to our calculations, ICCs for the $K$ shell have no resonances for all $Z$ and multipolarities.

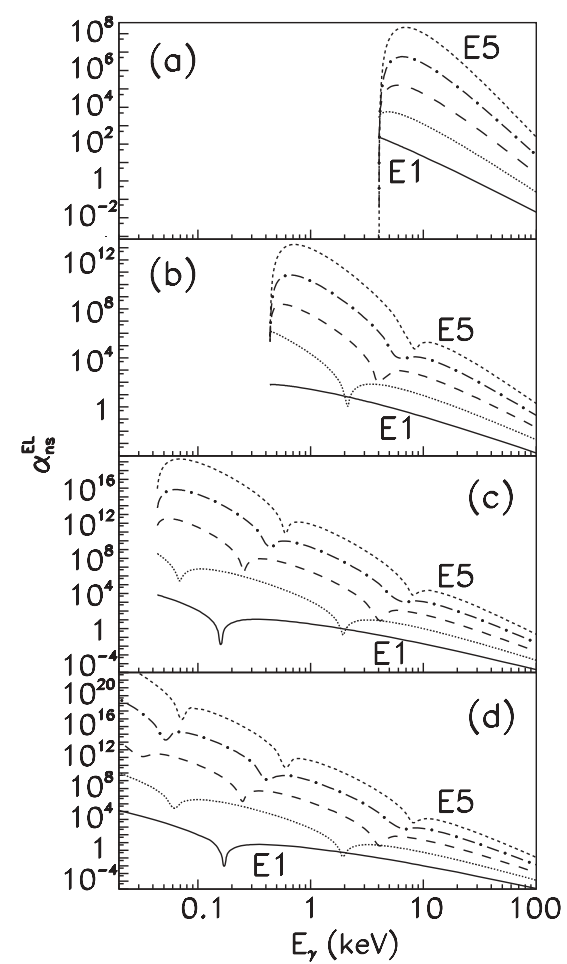

FIG. 1. ICCs versus the $\gamma$-ray energy $E_{\gamma}$ for the $n s$ shells of calcium $(Z=20)$ : (a), $K$ shell; (b), $L_{1}$ shell; (c), $M_{1}$ shell; (d), $N_{1}$ shell. Transitions marked as $E 1$ : solid; $E 2$ : dotted; $E 3$ : dashed; E4: chain; E5: small-size dashed lines.
As is seen in Fig. 1, there is only one resonance for the $L_{1}$ shell at E2-E5 transitions, but there are two resonances for the $M_{1}$ shell and three resonances for the $N_{1}$ shell. For various electric multipolarities, the positions of the resonances $E_{\gamma}^{(\text {res })}$ shift to the right as $L$ increases according to Eq. (12) but the general structure remains the same. For the $M_{1}$ and $N_{1}$ shells, new additional resonances arise at lower energies $E_{\gamma} \lesssim 1 \mathrm{keV}$. One can see also that Eq. (12) is justified in the case of the highest resonance $\left(2 \mathrm{keV} \lesssim E_{\gamma} \lesssim\right.$ $8 \mathrm{keV}$ ) not only for the $L_{1}$ shell but also for the higher $n s$ shells.

It should be emphasized that ICC for $E 1$ transition has one well-marked resonance for the $n s$ shells higher than $L_{1}$, while it was commonly assumed there was no resonance for $E 1$ transition. The resonance becomes deeper for higher shells. It is evident from Fig. 1 that the energy of $E 1$ resonances is approximately the same for all $n s$ shells with different $n$. One may anticipate that the $L_{1}$ shell has no $E 1$ resonance because the $L_{1}$ binding energy $\varepsilon_{L_{1}}=0.44 \mathrm{keV}$ for $Z=20$ exceeds the resonance energy $E_{\gamma}^{(\text {res })} \lesssim 0.2 \mathrm{keV}$ obtained for the higher $n s$ shells. However ICC for the $L_{1}$ shell of light elements $(Z \leqslant 11)$ with small binding energies has the $E 1$ resonance [see Fig. 5(a) below for $Z=11$ ].

For higher $Z$, resonances become less dominant. This is illustrated in Fig. 2 where ICCs for zirconium $(Z=40)$ for $E 1-E 5$ transitions and the high $N_{1}$ and $O_{1}$ shells are shown. One can see the same resonance structure for the $N_{1}$ shell but not so sharply as defined for $Z=20$. The $O_{1}$ shell has four resonances for $E 3-E 5$ transitions, two rather weak resonances for $E 2$ transition, and one well-defined resonance for $E 1$ transition. It should be noted that the resonances are visible in ICCs even for heavy elements as is shown in Fig. 3 for the $O_{1}$ shell of tungsten $(Z=74)$ where slight irregularities are remained for $E 2-E 5$ transitions.

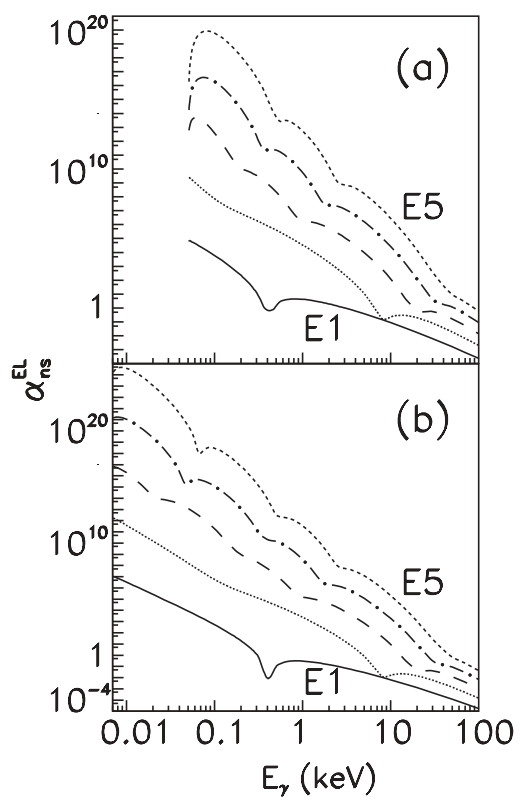

FIG. 2. ICCs versus the energy $E_{\gamma}$ for the $N_{1}$ (a) and $O_{1}$ (b) shells of zirconium $(Z=40)$. Transitions marked as $E 1$ : solid; $E 2$ : dotted; E3: dashed; $E$ 4: chain; $E$ 5: small-size dashed lines. 


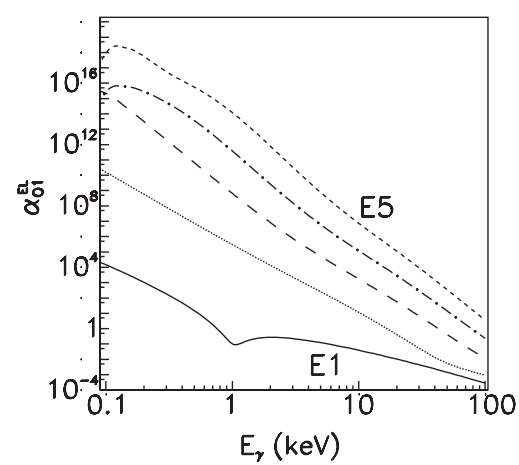

FIG. 3. ICCs for the $O_{1}$ shell of tungsten $(Z=74)$ at electric transitions. Transitions marked as E1: solid; $E 2$ : dotted; E3: dashed; E4: chain; E5: small-size dashed lines.

The ICC resonance at $E 1$ transition for the $n s$ shells with enough small $\varepsilon_{n s}$ exists for all elements up to fermium $(Z=$ 100). We see the well-defined $E 1$ resonance in Fig. 3 for the $O_{1}$ shell and in Fig. 4, where ICCs for the $N_{1}$ shell of elements with $20 \leqslant Z \leqslant 100$ are given. The $E 1$ resonance becomes less deep and wider as $Z$ increases, nevertheless there is a noticeable resonance even for fermium. The position of the resonance shifts to higher energies from $0.17 \mathrm{keV}$ for $Z=20$ to $3.2 \mathrm{keV}$ for $Z=100$. The resonance for $E 1$ transition is deeper than for other transitions (see Figs. 1 and 2). Because of this, the $E 1$ resonance still persists for fermium.

As mentioned above, Fig. 5(a) shows the $E 1$ resonance for the $L_{1}$ shell in a light element $(Z=11)$ at a low energy $E_{\gamma}^{(\text {res })}=$ $0.079 \mathrm{keV}$. This appears to be associated with the small binding energy $\varepsilon_{L_{1}}=0.066 \mathrm{keV}$ for the $L_{1}$ shell in the sodium atom. Note that the $E 1$ resonance in the $L_{1}$ shell of as light element as $\operatorname{Li}(Z=3)$ exists at $E_{\gamma}^{\text {(res) }}=0.015 \mathrm{keV}$ while $\varepsilon_{L_{1}} \approx 0.005 \mathrm{keV}$. In Fig. 5, the $E 1$ resonances are shown for various $n s$ shells of elements with $Z=11,37,74$, and 100 . One can see that the most considerable shift is between resonances for the $L_{1}$ and $M_{1}$ shells of sodium which equals $17 \%$. The shift between different $n s$ shells decreases with increasing nuclear charge.

We found expressions similar to Eq. (12) for the approximate resonance energy $E_{\gamma}^{(\mathrm{res})}$ for $E 1$ transition and the $n s$ shells. The expressions were obtained by the use of a fitting

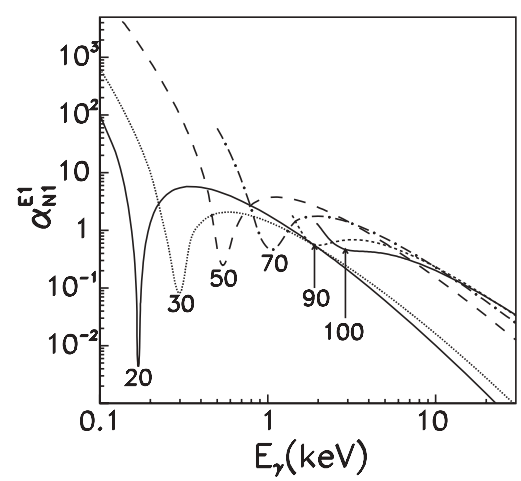

FIG. 4. ICCs for the $N_{1}$ shell of various elements at $E 1$ transition. Indicated under each minimum is the corresponding atomic number.

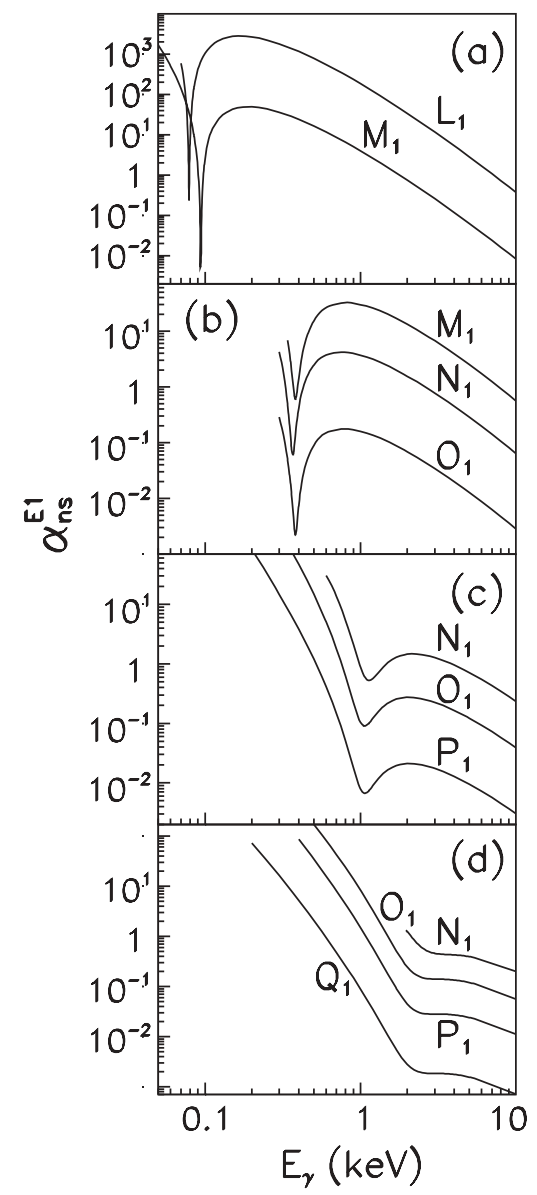

FIG. 5. ICCs in the $n s$ shells of representative elements for $E 1$ transition. (a), $Z=11$; (b), $Z=37$; (c), $Z=74$; (d), $Z=100$.

and may be written as follows

$$
\begin{aligned}
& E_{\gamma}^{(\mathrm{res})}(\mathrm{keV}) \approx \frac{Z^{1.28}}{273} \quad \text { for } \quad Z \leqslant 55, \\
& E_{\gamma}^{(\mathrm{res})}(\mathrm{keV}) \approx \frac{Z^{2.29}}{16470} \quad \text { for } \quad Z \geqslant 56 .
\end{aligned}
$$

The fitting was produced by the simplex search method [27]. To derive Eq. (13a), we adopted calculated values of $E_{\gamma}^{\text {(res) }}$ for 23 shells of 13 elements with $3 \leqslant Z \leqslant 55$. The relative root-mean-square (rms) error $\delta$ occurs to be equal $4.7 \%$. To obtain Eq. (13b), values of $E_{\gamma}^{\text {(res) }}$ for 23 shells of 10 elements with $56 \leqslant Z \leqslant 100$ were used. In this case, $\delta=5.5 \%$.

We also found the $E 1$ resonances for the $n p_{1 / 2}$ and $n p_{3 / 2}$ shells with $n \geqslant 3$. The resonances for the $M_{2}$ and $M_{3}$ shells were revealed for $13 \leqslant Z \leqslant 37$ where binding energies $\varepsilon_{M_{2}, M_{3}}$ are small enough. In Fig. 6, ICCs for $E 1$ transition and the $n p_{1 / 2}$ and $n p_{3 / 2}$ shells of $Z=15,36,74$, and 100 are displayed. Positions of the $E 1$ resonance for the $n p_{1 / 2}$ and $n p_{3 / 2}$ shells coincide for light and middle elements. This is demonstrated by the example of the $M_{2}$ and $M_{3}$ shells of $Z=15$ and the $N_{2}$ and $N_{3}$ shells of $Z=36$. There is no $E 1$ resonance for the $M_{2}$ and $M_{3}$ shells of $Z \geqslant 37$ because the binding energy of the shells increases, resulting in a degeneration of the resonance into a sharp rise of the ICCs 


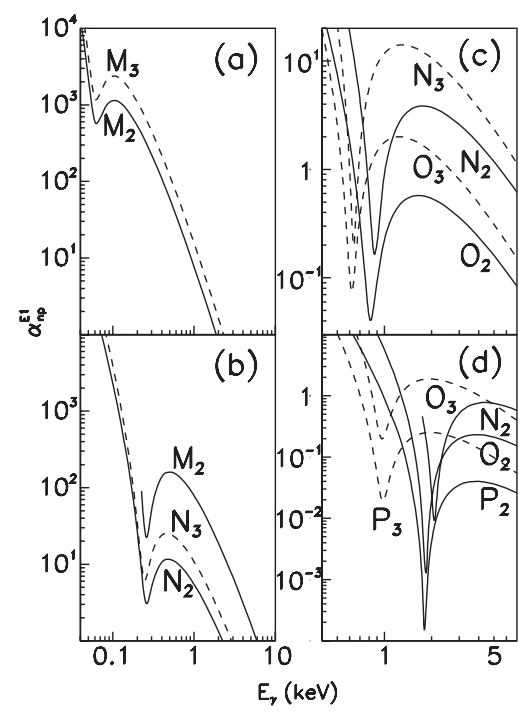

FIG. 6. ICCs in the $n p_{1 / 2}$ (solid) and $n p_{3 / 2}$ (dashed) shells of selected elements for $E 1$ transition. (a) $Z=15$; (b) $Z=36$; (c) $Z=74$; (d) $Z=100$.

starting from the energy threshold, i.e., from the subshell binding energy. However, for more heavy elements, the $E 1$ resonance arises in the higher $n p$ shells up to superheavy elements.

The resonance positions for shells with different $n$ are rather close, shifting slightly to the left as $n$ increases. For example, in the case of $Z=74, E_{\gamma}^{(\text {res })}=0.87 \mathrm{keV}$ for the $N_{2}$ shell and $E_{\gamma}^{(\text {res })}=0.82 \mathrm{keV}$ for the $O_{2}$ shell. For $Z=100, E_{\gamma}^{(\text {res })}=$ $2.08 \mathrm{keV}, 1.83 \mathrm{keV}$, and $1.80 \mathrm{keV}$ for the $N_{2}, O_{2}$, and $P_{2}$ shells, respectively. However, there is a large difference between values of $E_{\gamma}^{(\text {res })}$ for subshells of the relativistic $p$-doublet of heavy elements. For example, values of $E_{\gamma}^{(\text {res })}$ differ by more than $30 \%$ for the $\mathrm{N}_{2}$ and $\mathrm{N}_{3}$ subshells as well as for the $\mathrm{O}_{2}$ and $O_{3}$ subshells of $Z=74$ [Fig. 6(c)]. For $Z=100$, values of $E_{\gamma}^{(\text {res })}$ for the $O_{2}$ and $O_{3}$ subshells differ twice from one another as well as for the $P_{2}$ and $P_{3}$ subshells [Fig. 6(d)].

In Fig. 7, the $E 1$ resonances for the $N_{2}$ shell of representative elements from $Z=32$ to $Z=112$ are shown. One can see that, as distinct from the $n s$ shells, the resonance for the

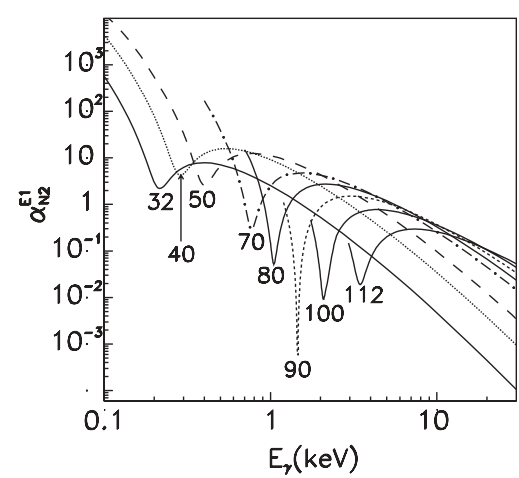

FIG. 7. ICCs for $E 1$ transition and the $N_{2}$ shell of various elements. Indicated under each minimum is the corresponding atomic number. $n p$ shells becomes deeper and more narrow with increasing $Z$ up to $Z \approx 90$. For $Z=90, \alpha_{N_{2}}^{E 1}$ falls more than three orders of magnitude at $E_{\gamma}^{(\text {res })}$. Then the resonances become wider and less deep. However, even for $Z=100$, the $E 1$ resonance for the $N_{2}$ shell is much deeper than for the $N_{1}$ shell (see Fig. 4).

Expressions for the approximate resonance energy for the $n p$ shells and $E 1$ transition obtained with the fitting have the following form

$$
\begin{aligned}
& E_{\gamma}^{(\mathrm{res})}(\mathrm{keV}) \approx \frac{Z^{1.54}}{1062} \quad \text { for } \quad Z \leqslant 55, \\
& E_{\gamma}^{(\mathrm{res})}(\mathrm{keV}) \approx \frac{Z^{1.76}}{2691} \quad \text { for } \quad Z \geqslant 56 .
\end{aligned}
$$

To obtain Eq. (14a), we adopted calculated values of $E_{\gamma}^{(\text {res })}$ for 27 shells of 14 elements with $13 \leqslant Z \leqslant 55$. The relative rms error $\delta$ occurs to be equal 5.4\%. For Eq. (14b), values of $E_{\gamma}^{(\text {res })}$ for 45 shells of 10 elements with $56 \leqslant Z \leqslant 100$ were used. In this case, $\delta=20 \%$. A large error is due to a large difference in $E_{\gamma}^{\text {(res) }}$ between the $p_{1 / 2}$ and $p_{3 / 2}$ electrons for heavy elements [see Figs. 6(c) and 6(d)].

As mentioned in Sec. I, Dragoun et al. [25] were the first who detected the $E 1$ resonance in ICC for the $5 d_{3 / 2}$ shell of protactinium $(Z=91)$. We found that ICCs for the $n d$ subshells with $n \geqslant 4$ have the $E 1$ resonance for all elements from $Z=39$ up to superheavy ones. It is seen in Fig. 8 where the resonances in the $N_{4}$ subshell for $39 \leqslant Z \leqslant 85$ (a) as well as in the $O_{4}$ subshell for $64 \leqslant Z \leqslant 112$ (b) are shown. All the resonances are located in the comparatively narrow $E_{\gamma}$ interval from 0.13 to $0.89 \mathrm{keV}$. The splitting of $E_{\gamma}^{(\text {res })}$ for the

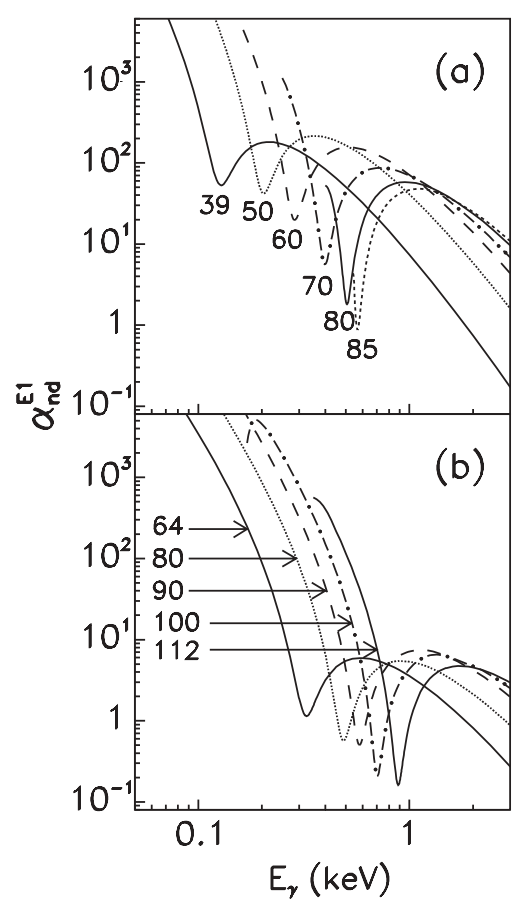

FIG. 8. ICCs for $E 1$ transition and the $N_{4}$ shell (a) as well as the $\mathrm{O}_{4}$ shell (b) of various elements. Indicated under each minimum in panel (a) or to the left of each curve in panel (b) is the corresponding atomic number. 


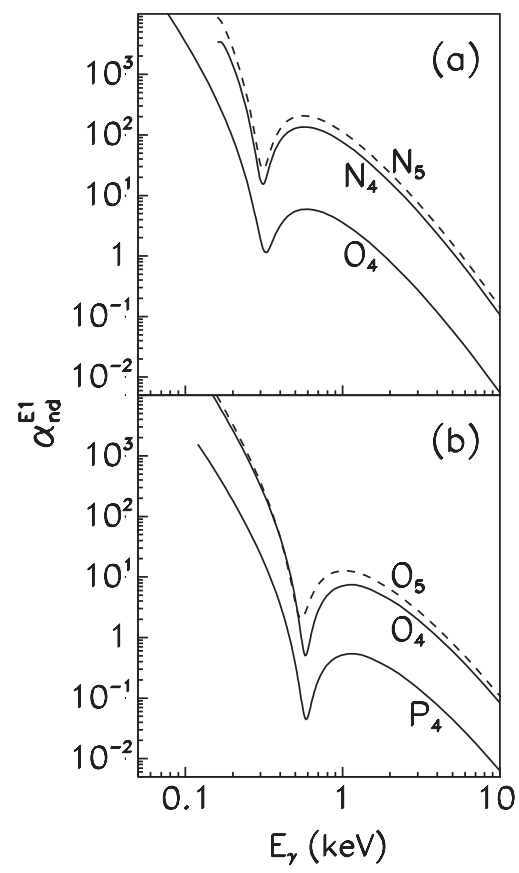

FIG. 9. ICCs at $E 1$ transition for the $d_{3 / 2}$ (solid) and $d_{5 / 2}$ (dashed) subshells of gadolinium $(Z=64)$ (a) as well as of thorium $(Z=90)(b)$.

$d_{3 / 2}$ and $d_{5 / 2}$ subshells is rather small as compared with the $p_{1 / 2}$ and $p_{3 / 2}$ splitting. This is shown in Fig. 9 where ICCs for the $N_{4}, N_{5}$, and $O_{4}$ of $Z=64$ (a) as well as for the $O_{4}$, $O_{5}$, and $P_{4}$ of $Z=90$ (b) are given. For $Z=100$, the splitting does not exceed $\sim 10 \%$ for the $O_{4}$ and $O_{5}$ subshells while for the $O_{2}$ subshell, $E_{\gamma}^{(\text {res })}$ is twice as large as for the $O_{3}$ subshell. Figure 9 shows also that $E_{\gamma}^{\text {(res) }}$ for the $n d$ shells does not practically depend on the principal quantum number $n$.

The approximate $E 1$ resonance energy for the $n d$ shells of all elements $39 \leqslant Z \leqslant 100$ can be written as follows:

$$
E_{\gamma}^{(\mathrm{res})}(\mathrm{keV}) \approx \frac{Z^{1.78}}{5191}
$$

In this case, values of $E_{\gamma}^{(\text {res })}$ for 34 shells of 15 elements with $39 \leqslant Z \leqslant 100$ were adopted. The relative rms error $\delta$ is equal to $3.8 \%$.

\section{PECULIARITIES OF RESONANCES}

Let us consider the origin of the ICC resonances in terms of conversion matrix elements and electron wave functions involved. The selection rules [see Eqs. (2), (3), and (5)] allow one to obtain readily the final electron states entering into the sum in Eq. (1) for ICC in various atomic subshells. The final electron states for conversion in the $s, p$, and $d$ shells are listed in Table I.

As is seen from Table I, for the $s$-conversion and $E L$ transitions, there are only two final states making up two components of a relativistic doublet with the same $\ell_{f}$ and total electron momenta $j_{1}=\ell_{f}-1 / 2$ and $j_{2}=\ell_{f}+1 / 2$. Because of the electron wave functions for states $\ell_{f} j_{1}$ and $\ell_{f} j_{2}$ are close to each other, especially for low $Z$, the relevant matrix elements $M_{n s}^{E L}\left(\ell_{f} j_{1}\right)$ and $M_{n s}^{E L}\left(\ell_{f} j_{2}\right)$ for a specific $n$ and $L$ are approximately equal in magnitude, being usually opposite in sign. The latter is not essential because ICC includes squared matrix elements. An important point is that both of the matrix elements change their signs at the approximately same energy $E_{\gamma}$ one or more times. The resonance occurs in the vicinity of this energy.

Figure 10 shows the partial matrix elements $M_{n s}^{E L}\left(\kappa_{f}\right) \equiv$ $M_{n s}^{E L}\left(\ell_{f} j_{f}\right)$ for conversion in the $N_{1}$ shell of calcium for final states $h_{9 / 2}\left(\kappa_{f}=5\right)$ and $h_{11 / 2}\left(\kappa_{f}=-6\right)$ entering into $E 5$ transition in the vicinity of resonance energies $E_{\gamma}^{\text {(res) }}=$ $0.0705 \mathrm{keV}(\mathrm{a}), 0.594 \mathrm{keV}(\mathrm{b})$, and $8.18 \mathrm{keV}(\mathrm{c})$. As it is visible, the matrix elements $M_{N_{1}}^{E 5}\left(h_{9 / 2}\right)$ and $M_{N_{1}}^{E 5}\left(h_{11 / 2}\right)$ are close to each other in absolute magnitude and both of them change sign three times, that is, have three roots, associated with three resonances in $\alpha_{N_{1}}^{E 5}\left(E_{\gamma}\right)$ demonstrated in Fig. 1.

The same situation is displayed in Fig. 11(a). Matrix elements for ICC in the $L_{1}$ shell of sodium at $E 2$ transition become equal to zero only once at $E_{\gamma}=0.643 \mathrm{keV}$ for the $d_{3 / 2}$ final state and at $E_{\gamma}=0.636 \mathrm{keV}$ for the $d_{5 / 2}$ final state. Because of this, the $E_{\gamma}$ dependence of ICC $\alpha_{L_{1}}^{E 2}\left(E_{\gamma}\right)$ has a resonance at the midpoint $E_{\gamma}^{(\mathrm{res})}=0.638 \mathrm{keV}$ as is shown

TABLE I. Final electron states involved in ICC for conversion in the $i$-th subshell.

\begin{tabular}{|c|c|c|c|c|c|}
\hline $\begin{array}{l}\ell_{i} j_{i} \\
\kappa_{i} \\
\tau L\end{array}$ & $\begin{array}{c}s_{1 / 2} \\
-1\end{array}$ & $\begin{array}{c}p_{1 / 2} \\
+1\end{array}$ & $\begin{array}{c}p_{3 / 2} \\
-2\end{array}$ & $\begin{array}{c}d_{3 / 2} \\
+2\end{array}$ & $\begin{array}{c}d_{5 / 2} \\
-3\end{array}$ \\
\hline$E 1$ & $p_{1 / 2}, p_{3 / 2}$ & $s_{1 / 2}, d_{3 / 2}$ & $s_{1 / 2}, d_{3 / 2}, d_{5 / 2}$ & $p_{1 / 2}, p_{3 / 2}, f_{5 / 2}$ & $p_{3 / 2}, f_{5 / 2}, f_{7 / 2}$ \\
\hline$E 2$ & $d_{3 / 2}, d_{5 / 2}$ & $p_{3 / 2}, f_{5 / 2}$ & $p_{1 / 2}, p_{3 / 2}, f_{5 / 2}, f_{7 / 2}$ & $s_{1 / 2}, d_{3 / 2}, d_{5 / 2}, g_{7 / 2}$ & $s_{1 / 2}, d_{3 / 2}, d_{5 / 2}, g_{7 / 2}, g_{9 / 2}$ \\
\hline$E 3$ & $f_{5 / 2}, f_{7 / 2}$ & $d_{5 / 2}, g_{7 / 2}$ & $d_{3 / 2}, d_{5 / 2}, g_{7 / 2}, g_{9 / 2}$ & $p_{3 / 2}, f_{5 / 2}, f_{7 / 2}, h_{9 / 2}$ & $p_{1 / 2}, p_{3 / 2}, f_{5 / 2}, f_{7 / 2}, h_{9 / 2}, h_{11 / 2}$ \\
\hline$E 4$ & $g_{7 / 2}, g_{9 / 2}$ & $f_{7 / 2}, h_{9 / 2}$ & $f_{5 / 2}, f_{7 / 2}, h_{9 / 2}, h_{11 / 2}$ & $d_{5 / 2}, g_{7 / 2}, g_{9 / 2}, i_{11 / 2}$ & $d_{3 / 2}, d_{5 / 2}, g_{7 / 2}, g_{9 / 2}, i_{11 / 2}, i_{13 / 2}$ \\
\hline$E 5$ & $h_{9 / 2}, h_{11 / 2}$ & $g_{9 / 2}, i_{11 / 2}$ & $g_{7 / 2}, g_{9 / 2}, i_{11 / 2}, i_{13 / 2}$ & $f_{7 / 2}, h_{9 / 2}, h_{11 / 2}, j_{13 / 2}$ & $f_{5 / 2}, f_{7 / 2}, h_{9 / 2}, h_{11 / 2}, j_{13 / 2}, j_{15 / 2}$ \\
\hline$M 1$ & $s_{1 / 2}, d_{3 / 2}$ & $p_{1 / 2}, p_{3 / 2}$ & $p_{1 / 2}, p_{3 / 2}, f_{5 / 2}$ & $s_{1 / 2}, d_{3 / 2}, d_{5 / 2}$ & $d_{3 / 2}, d_{5 / 2}, g_{7 / 2}$ \\
\hline$M 2$ & $p_{3 / 2}, f_{5 / 2}$ & $d_{3 / 2}, d_{5 / 2}$ & $s_{1 / 2}, d_{3 / 2}, d_{5 / 2}, g_{7 / 2}$ & $p_{1 / 2}, p_{3 / 2}, f_{5 / 2}, f_{7 / 2}$ & $p_{1 / 2}, p_{3 / 2}, f_{5 / 2}, f_{7 / 2}, h_{9 / 2}$ \\
\hline$M 3$ & $d_{5 / 2}, g_{7 / 2}$ & $f_{5 / 2}, f_{7 / 2}$ & $p_{3 / 2}, f_{5 / 2}, f_{7 / 2}, h_{9 / 2}$ & $d_{3 / 2}, d_{5 / 2}, g_{7 / 2}, g_{9 / 2}$ & $s_{1 / 2}, d_{3 / 2}, d_{5 / 2}, g_{7 / 2}, g_{9 / 2}, i_{11 / 2}$ \\
\hline$M 4$ & $f_{7 / 2}, h_{9 / 2}$ & $g_{7 / 2}, g_{9 / 2}$ & $d_{5 / 2}, g_{7 / 2}, g_{9 / 2}, i_{11 / 2}$ & $f_{5 / 2}, f_{7 / 2}, h_{9 / 2}, h_{11 / 2}$ & $p_{3 / 2}, f_{5 / 2}, f_{7 / 2}, h_{9 / 2}, h_{11 / 2}, j_{13 / 2}$ \\
\hline M5 & $g_{9 / 2}, i_{11 / 2}$ & $h_{9 / 2}, h_{11 / 2}$ & $f_{7 / 2}, h_{9 / 2}, h_{11 / 2}, j_{13 / 2}$ & $g_{7 / 2}, g_{9 / 2}, i_{11 / 2}, i_{13 / 2}$ & $d_{5 / 2}, g_{7 / 2}, g_{9 / 2}, i_{11 / 2}, i_{13 / 2}, k_{15 / 2}$ \\
\hline
\end{tabular}




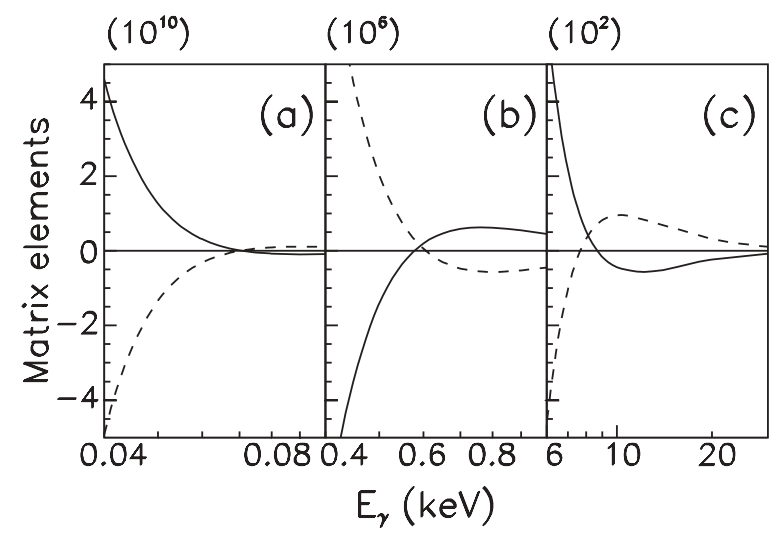

FIG. 10. Partial matrix elements entering into ICC in the $N_{1}$ shell of calcium $(Z=20)$ for $E 5$ transition in vicinities of resonances. $M_{N_{1}}^{E 5}\left(h_{9 / 2}\right)\left(\kappa_{f}=5\right)$ : solid; $M_{N_{1}}^{E 5}\left(h_{11 / 2}\right)\left(\kappa_{f}=-6\right)$ : dashed. In panels (a), (b), and (c), values of matrix elements should be multiplied by $10^{10}, 10^{6}$, and $10^{2}$, respectively.

in Fig. 12 where the corresponding ICCs are presented. Consequently, a vanishing of conversion matrix elements is a necessary condition for the occurrence of the ICC resonance. However, relevant matrix elements do not need to be equal to each other. For example, there are resonances in ICC in the $M_{2}$ and $M_{3}$ shells of zinc $(Z=30)$ at $E 1$ transition while as is seen from Figs. 11(b) and 11(c), matrix elements for various final states involved in $\alpha^{E 1}$ are rather different. For the $M_{2}$ shell, the resonance point $E_{\gamma}^{(\text {res })}=0.18 \mathrm{keV}$ (see Fig. 12) and just at this energy the larger matrix element $M_{M_{2}}^{E 1}\left(d_{3 / 2}\right)$ equals to zero. For the $M_{3}$ shell, we see from Figs. 11 and 12 that $E_{\gamma}^{(\text {res })}=$ $0.18 \mathrm{keV}$ which approximately coincides with the points of vanishing two matrix elements $M_{M_{3}}^{E 1}\left(d_{3 / 2}\right)$ and $M_{M_{3}}^{E 1}\left(d_{5 / 2}\right)$ at

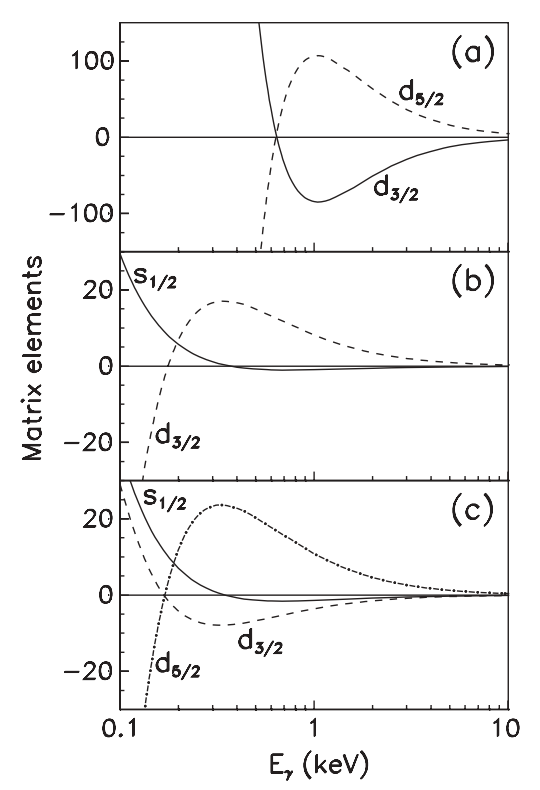

FIG. 11. Partial matrix elements $M_{i}^{E L}\left(\ell_{f} j_{f}\right)$ in the vicinities of resonances for ICCs in the $L_{1}$ shell of sodium $(Z=11)$ at the $E 2$ transition (a) as well as in the $M_{2}$ shell (b) and the $M_{3}$ shell (c) of zinc $(Z=30)$ at the $E 1$ transition.

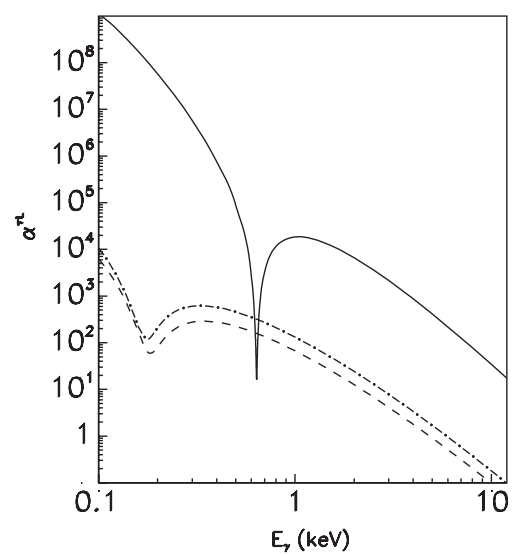

FIG. 12. Resonances in ICCs. Solid line: the $L_{1}$ shell of sodium ( $Z=11$ ) for the $E 2$ transition; dashed: the $M_{2}$ subshell of zinc ( $Z=30)$ at the $E 1$ transition; chain: the $M_{3}$ subshell of zinc at the $E 1$ transition.

$E_{\gamma}=0.17 \mathrm{keV}$ while a magnitude of the third component $M_{M_{3}}^{E 1}(s)$ does not vanish in the vicinity of the point.

In contrast, the ICC has no resonances when matrix elements which change sign are much smaller in magnitude than others of constant signs or all of them do not change signs. Such cases are presented in Table II for zinc. One may see that in the case of the $M_{2}$ shell and $E 2$ transition, $M_{M_{2}}^{E 2}\left(f_{5 / 2}\right)$ changes sign but this matrix element is two orders of magnitude smaller at low energies than $M_{M_{2}}^{E 2}\left(p_{3 / 2}\right)$ which has a constant sign. The same situation occurs in the case of the $M_{1}$ shell and $M 1$ transition: the alternating-sign $M_{M_{1}}^{M 1}\left(d_{3 / 2}\right)$ is two orders of magnitude smaller than $M_{M_{1}}^{M 1}\left(s_{1 / 2}\right)$ which does not change sign. For the magnetic transitions and conversion in the $n p$ and $n d$ shells involving final states with the same $\ell_{f}$ like electric transitions for the $s$ shells (see Table I), neither matrix element changes sign as shown in Table II for $M 1$ transition and the $M_{2}$ shell. For this reason, ICCs for magnetic transitions have no resonances.

Strong resonances in ICC for the $n s$ shells of light elements at $E 2-E 5$ transitions are attenuated as $Z$ increases because the difference between two matrix elements with common $\ell_{f} \geqslant 2$ becomes more and more considerable, the largest matrix element (or sometimes both of them) being sign constant. In Fig. 13, matrix elements for the $O_{1}$ shell of tungsten are given for $E 1$ and $E 2$ transitions. As is seen, matrix elements for $E 1$ transition $M_{O_{1}}^{E 1}\left(p_{1 / 2}\right)$ and $M_{O_{1}}^{E 1}\left(p_{3 / 2}\right)$ are still rather close to each other, the first changes sign at $0.83 \mathrm{keV}$ and the second at $1.14 \mathrm{keV}$. The relevant ICC has the $E 1$ resonance at the middle point $E_{\gamma}^{(\mathrm{res})}=1.06 \mathrm{keV}$ [see Fig. 5(c)]. However, matrix elements entering into ICC for $E 2$ transition $M_{O_{1}}^{E 2}\left(d_{3 / 2}\right)$ and $M_{O_{1}}^{E 2}\left(d_{5 / 2}\right)$ differ more than an order of magnitude, the largest $M_{O_{1}}^{E 2}\left(d_{3 / 2}\right)$ being positive in the whole energy range under consideration. So ICCs of $E 2$ transitions in the $O_{1}$ shell for $Z=74$ have no resonances at $E_{\gamma} \lesssim 10 \mathrm{keV}$. A slight minimum at $E_{\gamma} \approx 60 \mathrm{keV}$, which is visible in Fig. 3, comes from change sign of the smaller matrix element $M_{O_{1}}^{E 2}\left(d_{5 / 2}\right)$.

It is interesting to compare the formation region of ICC at a resonance energy with that outside the resonance energy 
TABLE II. Matrix elements $M_{i}^{\tau L}\left(\ell_{f} j_{f}\right)$ for ICCs with no resonance, $Z=30$. The decimal order of magnitudes is given parenthetically.

\begin{tabular}{|c|c|c|c|c|c|c|}
\hline \multirow[t]{2}{*}{$\ell_{f} j_{f} E_{\gamma}(\mathrm{keV})$} & \multicolumn{2}{|c|}{$M_{M_{2}}^{E 2}\left(\ell_{f} j_{f}\right)$} & \multicolumn{2}{|c|}{$M_{M_{1}}^{M 1}\left(\ell_{f} j_{f}\right)$} & \multicolumn{2}{|c|}{$M_{M_{2}}^{M 1}\left(\ell_{f} j_{f}\right)$} \\
\hline & $p_{3 / 2}$ & $f_{5 / 2}$ & $s_{1 / 2}$ & $d_{3 / 2}$ & $p_{1 / 2}$ & $p_{3 / 2}$ \\
\hline 0.14 & $-4.20(5)$ & $-5.74(3)$ & $-4.39(2)$ & $1.16(-1)$ & $-1.26(2)$ & $-2.08(1)$ \\
\hline 0.20 & $-1.75(5)$ & $-4.37(3)$ & $-2.58(2)$ & $-3.07(-1)$ & $-7.51(1)$ & $-1.24(1)$ \\
\hline 0.30 & $-6.43(4)$ & $-2.06(3)$ & $-1.42(2)$ & $-4.66(-1)$ & $-4.12(1)$ & $-6.82(0)$ \\
\hline 0.40 & $-3.14(4)$ & $-9.92(2)$ & $-9.29(1)$ & $-4.49(-1)$ & $-2.68(1)$ & $-4.44(0)$ \\
\hline 0.60 & $-1.14(4)$ & $-2.60(2)$ & $-5.09(1)$ & $-3.52(-1)$ & $-1.46(1)$ & $-2.42(0)$ \\
\hline 1.00 & $-3.18(3)$ & 7.61(0) & $-2.38(1)$ & $-2.10(-1)$ & $-6.74(0)$ & $-1.12(0)$ \\
\hline 2.00 & $-5.55(2)$ & $3.39(1)$ & $-8.47(0)$ & $-6.96(-2)$ & $-2.35(0)$ & $-3.91(-1)$ \\
\hline 3.00 & $-1.99(2)$ & $2.13(1)$ & $-4.63(0)$ & $-2.27(-2)$ & $-1.26(0)$ & $-2.10(-1)$ \\
\hline 5.00 & $-5.46(1)$ & $9.52(0)$ & $-2.16(0)$ & $8.71(-3)$ & $-5.75(-1)$ & $-9.58(-2)$ \\
\hline 8.00 & $-1.65(1)$ & $4.01(0)$ & $-1.07(0)$ & $1.97(-2)$ & $-2.78(-1)$ & $-4.63(-2)$ \\
\hline 10.00 & $-9.38(0)$ & $2.58(0)$ & $-7.69(-1)$ & $2.12(-2)$ & $-1.96(-1)$ & $-3.27(-2)$ \\
\hline 15.00 & $-3.34(0)$ & $1.12(0)$ & $-4.21(-1)$ & $2.05(-2)$ & $-1.48(-1)$ & $-2.46(-2)$ \\
\hline 20.00 & $-1.61(0)$ & $6.04(-1)$ & $-2.75(-1)$ & $1.86(-2)$ & $-6.64(-1)$ & $-1.11(-2)$ \\
\hline
\end{tabular}

range. It has been shown in Ref. [28] that usually the inner part of an atom is of major importance in the formation of ICC in all atomic shells, including the most outer ones for various transitions in the wide energy range.

Let us consider the $r$ dependence of the radial matrix element $R_{n s}^{E L}\left(\ell_{f} j_{f}\right)$. For $E L$ transitions, the radial integral $R_{3, \Lambda=L}$ [Eq. (9)] makes a major contribution to the matrix element, the imaginary part of $R_{3, \Lambda=L}$ being usually several orders of magnitude larger than the real part. So we consider

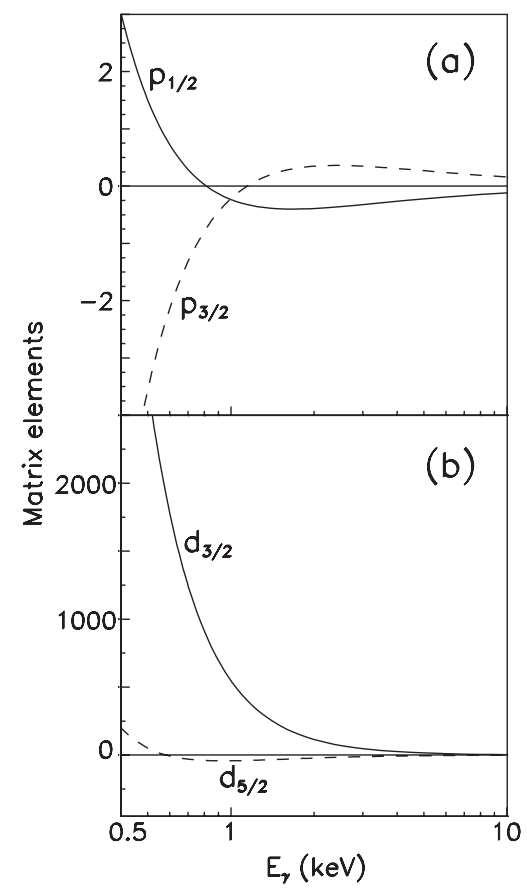

FIG. 13. Partial matrix elements $M_{O_{1}}^{E L}\left(\ell_{f} j_{f}\right)$ for ICCs in the $O_{1}$ shell of tungsten $(Z=74)$ for $E 1$ (a) and $E 2$ (b) transitions. the $r$ dependence of the integral

$$
\begin{aligned}
\operatorname{Im} R_{3, \Lambda=L}(r)= & (-1)^{L+1} \int_{0}^{r}\left[G_{i}\left(r^{\prime}\right) G_{f}\left(E_{k}, r^{\prime}\right)\right. \\
& \left.+F_{i}\left(r^{\prime}\right) F_{f}\left(E_{k}, r^{\prime}\right)\right] \\
& \times\left\{\begin{array}{cc}
j_{L}\left(k r^{\prime}\right) \frac{j_{-(L+1)}\left(k R_{0}\right)}{j_{L}\left(k R_{0}\right)} & \text { for } r^{\prime} \leqslant R_{0}, \\
j_{-(L+1)}\left(k r^{\prime}\right) & \text { for } r^{\prime}>R_{0} .
\end{array}\right.
\end{aligned}
$$

The integral of Eq. (16) for the $N_{1}$ shell of calcium and $E 2$ transition is demonstrated in Fig. 14 together with large components of discrete $G_{i}(r)$ and continuum $G_{f}\left(E_{k}, r\right)$ wave functions versus the distance $r$ in atomic units $(1$ a.u. $=$ $52919 \mathrm{fm})$. We present the continuum wave function and the matrix element only for $d_{5 / 2}(\kappa=-3)$ because both states $d_{3 / 2}$ and $d_{5 / 2}$ involving in $\alpha_{N_{1}}^{E 2}$ are close to each other in this case. Figures 14(a)-14(c) and 14(d)-14(f) refer to two resonance energies $E_{\gamma}^{(\text {res })}=0.062 \mathrm{keV}$ and $E_{\gamma}^{(\text {res })}=1.942 \mathrm{keV}$, respectively (see Fig. 1). Figures $14(\mathrm{~g})-14(\mathrm{i})$ refer to $E_{\gamma}=$ $10 \mathrm{keV}$ which is outside the resonance regions.

Figure 14 makes it clear that in the resonance cases, the matrix elements under consideration, and, consequently, corresponding ICCs, are formed in a large atomic region revealing oscillations with large and small amplitudes as is shown in panels (c) and (f). The negative minimum of the curve in panel (f) exceeds the asymptotic value of the integral [Eq. (16)] by a factor of 46 . The formation region radius, that is, the distance where the integral reaches its asymptotic value, equals to several atomic units which correlates with the radius of the valence $4 s$ shell. On the contrary, as is shown in panel (i), outside the resonance energy range, the matrix element attains an asymptotic value smoothly with no drastic changes. In this case, the formation region radius is an order of magnitude smaller, that is the ICC forms in the inner part of the atom.

Besides, it should be noted that in the vicinity of $E_{\gamma}^{(\text {res })}$, the first principal maximum of the continuum wave function $G_{f}$ is placed approximately at the same distance $r$ as one 


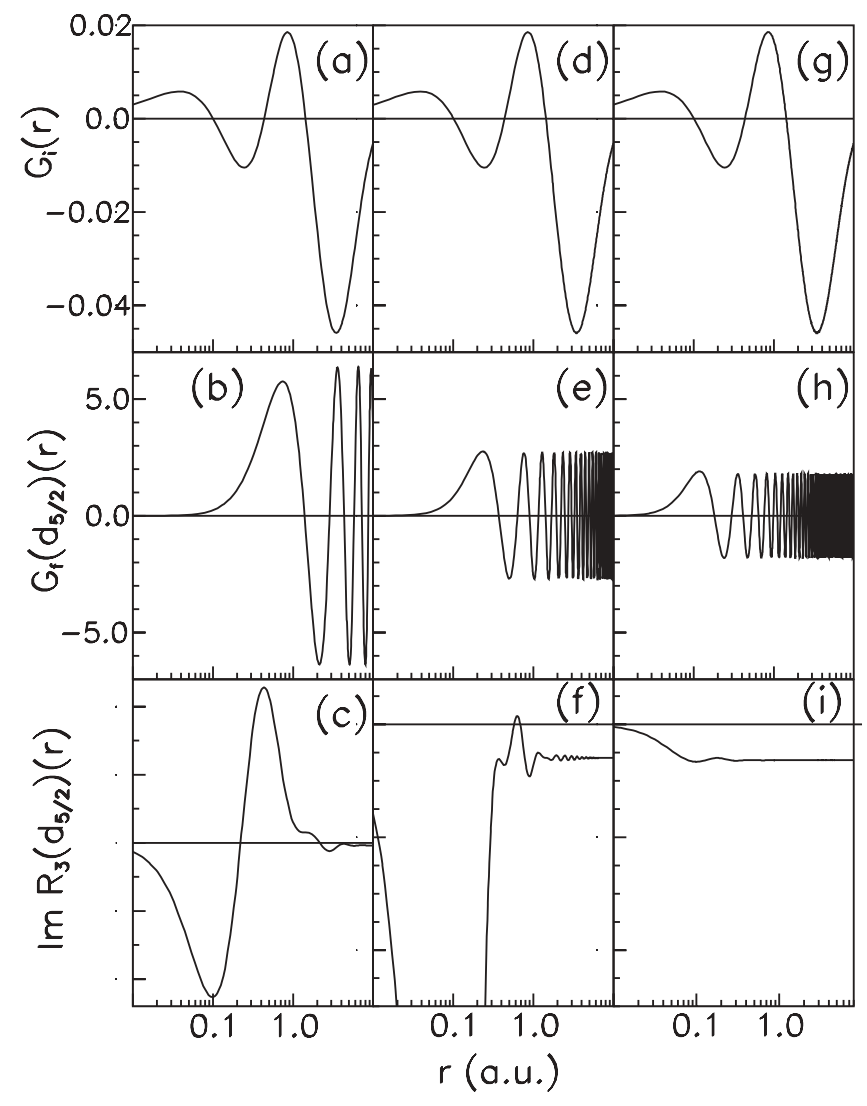

FIG. 14. Discrete $G_{i}(r)$ and continuum $G_{f}\left(E_{k}, r\right)$ wave functions along with partial radial matrix elements with a variable upper limit [Eq. (16)] for the $d_{5 / 2}$ final state involving in ICC for the $N_{1}$ shell of calcium $(Z=20)$ and for $E 2$ transition in vicinities of resonances at $E_{\gamma}^{(\text {res })}=0.062 \mathrm{keV}[(\mathrm{a})-(\mathrm{c})]$ and $E_{\gamma}^{(\text {res })}=1.942 \mathrm{keV}[(\mathrm{d})-(\mathrm{f})]$ as well as outside the resonance ragions at $E_{\gamma}=10 \mathrm{keV}$ [(g)-(i)]. In panels (c), (f), and (i), the matrix elements are displayed at different scales, so only zero is marked off by a solid horizontal line.

of the extrema (positive or negative) of the relevant discrete function $G_{i}$. As it is seen in Fig. 14, at the energy of the first (lowest) resonance, the position of the principal maximum of $G_{f}$ coincides with the position of the third extremum of $G_{i}$ [Figs. 14(a) and 14(b)] while at the energy of the second resonance, the principal maximum of $G_{f}$ coincides with the position of the second extremum of $G_{i}$ [Figs. 14(d) and 14(e)].

The same regularity is clearly visible also in Fig. 15 where discrete and continuum wave functions entering into the matrix element $M_{N_{1}}^{E 5}\left(h_{9 / 2}\right)$ for the $N_{1}$ shell of calcium at $E 5$ transition are presented. The continuum wave functions are displayed for three values of $E_{\gamma}^{(\text {res })}$ (see Fig. 1). The principal maximum of the continuum wave function $G_{f}\left(h_{9 / 2}\right)$ is close to the principal (the forth) extremum of $G_{N_{1}}$ for the lowest resonance of $E_{\gamma}^{(\text {res })}=0.0705 \mathrm{keV}$ at a distance of $r=3-3.5$ a.u. from the atomic center. The maximum coincides rather well with the third extremum of $G_{N_{1}}$ for the middle resonance of $E_{\gamma}^{\text {(res) }}=0.594 \mathrm{keV}$ at $r=0.9$ a.u., and with the second extremum of $G_{N_{1}}$ for the highest resonance of $E_{\gamma}^{(\text {res })}=8.18 \mathrm{keV}$ at $r=0.25$ a.u. Such relation between extrema of the discrete and continuum functions is likely to be optimal for the occurrence of the resonances in ICCs.

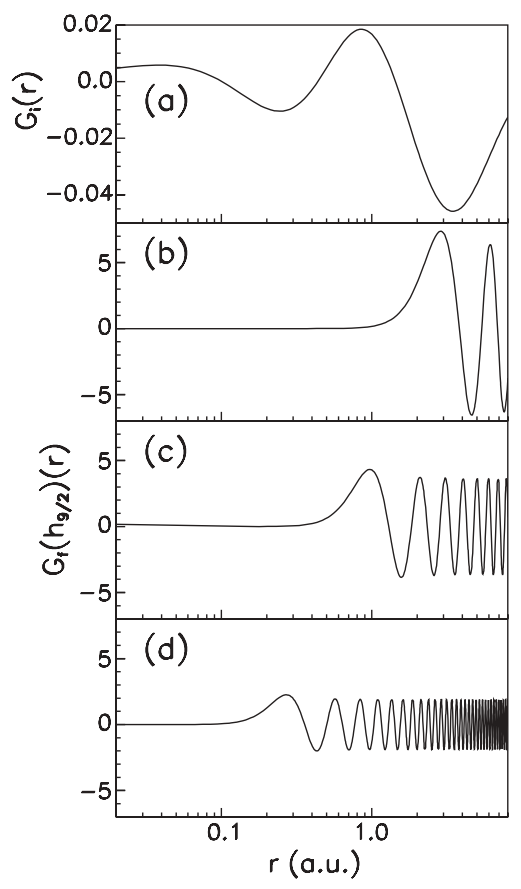

FIG. 15. Discrete $G_{i}(r)$ (a) and continuum $G_{f}\left(h_{9 / 2}\right)(r)$ wave functions entering into the ICC in the $N_{1}$ shell of calcium $(Z=20)$ for $E 5$ transition at three resonance energies $E_{\gamma}^{(\text {res })}=0.0705 \mathrm{keV}$ (b), $0.594 \mathrm{keV}$ (c), and $8.18 \mathrm{keV}$ (d) versus a distance from the atomic center.

\section{EXPERIMENTAL VERIFICATION OF RESONANCES}

To verify the existence of the resonances, the ENSDF [29] database was searched for suitable candidates. We only considered pure $E 1-E 5$ multipolarities and $n s$ atomic shells. Equations (12) and (13) were used as a guide to predict the position of the resonances. Since the resonant energies are usually rather low, in most cases only the relative subshell conversion electron intensities are known. The fact that no $E 1$ transition was found is not surprising as the resonance is very sharp and is expected at very low energies. For $E 2-E 5$ transitions, resonances are not expected in the $L_{2}$ shell, therefore the experimental $L_{1} / L_{2}$ subshell ratio is a suitable measure to test the presence of resonant behavior in the $L_{1}$ shell.

Table III contains 13 nuclear transitions for which the $L_{1} / L_{2}$ subshell ICC ratio has been measured [30-42]. The experimental values are compared with theoretical ones. The uncertainty on the theoretical values only contain contributions from the uncertainty on the $\gamma$-ray energy. The table contains nine $E 2$ and four $E 3$ transitions with atomic numbers ranging from 47 to 88 . All $\alpha_{L_{1}}^{E L} / \alpha_{L_{2}}^{E L}$ ratios listed in Table III have a pronounced resonance relating to nonmonotonic behavior of $\alpha_{L_{1}}^{E L}$. Considering all 13 cases, the average ratio of the experimental $\alpha_{L_{1}}^{E L} / \alpha_{L_{2}}^{E L}$ values to the theoretical ones is $1.3 \pm 0.6$. This was obtained with the bootstrap method [43], which does not require the uncertainties to determine the mean value of a data set. By excluding the ${ }^{142} \mathrm{Sm}$ and ${ }^{191} \mathrm{Ir}$ data points, the average value is $1.17 \pm 0.15$, indicating a satisfactory agreement between theory and experiment. 
TABLE III. Experimental and theoretical values of the $L_{1} / L_{2}$ subshell conversion coefficient ratios for transitions with energies near the expected position of resonances.

\begin{tabular}{|c|c|c|c|c|c|}
\hline \multirow[t]{2}{*}{ Nucleus } & \multicolumn{2}{|c|}{ Transition } & \multicolumn{3}{|c|}{$\alpha_{L_{1}} / \alpha_{L_{2}}$} \\
\hline & $E_{\gamma}(\mathrm{keV})$ & $\tau L$ & Experiment & Refs. & Calculated \\
\hline${ }_{47}^{105} \mathrm{Ag}$ & $25.482^{\mathrm{a}}$ & $E 3$ & 0.002 & [30] & 0.0024024 \\
\hline${ }_{62}^{142} \mathrm{Sm}$ & 24.13 & $E 2$ & 0.2035 & [31] & 0.0054612 \\
\hline${ }_{63}^{152} \mathrm{Eu}$ & 39.7510 & $E 3$ & 0.022 & [32] & 0.011012 \\
\hline${ }_{70}^{167} \mathrm{Yb}$ & 28.8808 & $E 2$ & 0.015 & [33] & 0.011131 \\
\hline${ }_{71}^{170} \mathrm{Lu}$ & 44.5210 & $E 2$ & 0.015 & [34] & 0.011625 \\
\hline${ }_{77}^{191} \mathrm{Ir}$ & 47.053 & $E 2$ & 0.06342 & [35] & 0.015471 \\
\hline${ }_{79}^{195} \mathrm{Au}$ & 56.803 & $E 3$ & 0.0182 & [36] & 0.024031 \\
\hline${ }_{79}^{196} \mathrm{Au}$ & 84.662 & $E 3$ & 0.040 & [37] & 0.027501 \\
\hline${ }_{80}^{198} \mathrm{Hg}$ & 47.745 & $E 2$ & 0.0258 & [38] & 0.018761 \\
\hline${ }_{83}^{206} \mathrm{Bi}$ & 59.90818 & $E 2$ & 0.0231 & [39] & 0.023501 \\
\hline${ }_{87}^{221} \mathrm{Fr}$ & 38.51 & $E 2$ & 0.03523 & [40] & 0.028601 \\
\hline${ }_{88}^{223} \mathrm{Ra}$ & 61.44120 & $E 2$ & 0.0263 & [41] & 0.030341 \\
\hline${ }_{88}^{224} \mathrm{Ra}$ & 84.3733 & $E 2$ & 0.0392 & [42] & 0.03716 \\
\hline
\end{tabular}

${ }^{\mathrm{a} A c c o r d i n g}$ to our notation, $25.482 \equiv 25.48 \pm 0.02,0.0024024 \equiv 0.002402 \pm 0.000004$, etc.

Figure 16 compares the experimental $\alpha_{L_{1}}^{E 3} / \alpha_{L_{2}}^{E 3}$ values for silver $(Z=47)$ and gold $(Z=79)$ with theoretical ratios. We can see well-marked resonances in the ICC ratios for $E 3$

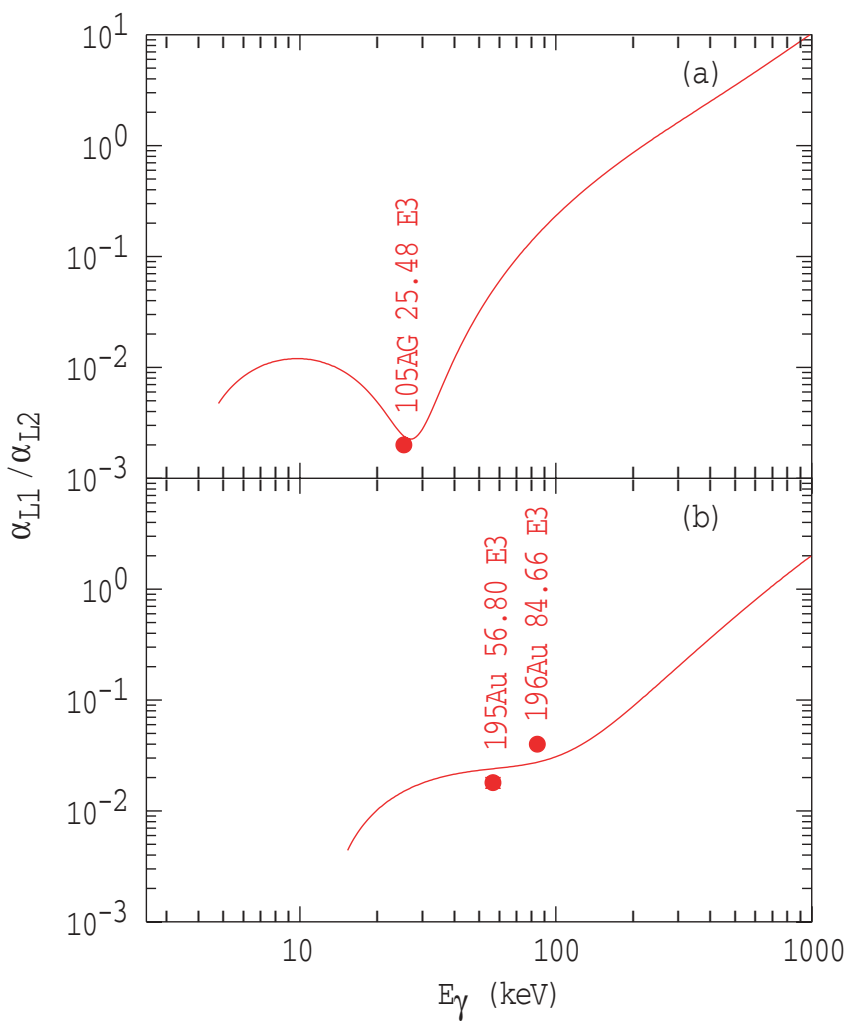

FIG. 16. (Color online) $L_{1} / L_{2}$ conversion coefficient ratios of $E 3$ transitions (a) in silver $(Z=47)$ and (b) in gold $(Z=79)$ nuclei. Experimental data are taken from Refs. [30,36,37]. Theoretical ratios are also shown as lines. transitions as well as a good agreement with experimental values in the vicinity of the resonances.

\section{CONCLUSIONS}

(i) Additional unknown resonance minima have been found in the $E_{\gamma}$ dependence of ICC at $E 2-E 5$ transitions for the $n s$ shells with $n \geqslant 2$ at low $\gamma$-ray energies. It is shown that ICCs may have up to four resonances for outer shells.

(ii) Resonances in ICCs at the $E 1$ transition have been discovered for the $n s$ shells from the $L_{1}$ shell of light elements to the $Q_{1}$ shell of heavy elements up to $Z=100$.

(iii) Resonances in ICCs at $E 1$ transition have been also found for the $n p$ shells with $n \geqslant 3$ and $n d$ shells with $n \geqslant 4$ of elements from light up to superheavy ones. All $E 1$ resonances occur at very low energies, less than several $\mathrm{keV}$. However, as is mentioned in Sec. I, these are the low-energy transitions which receive considerable interest currently.

(iv) In the case of $E 1$ transition, simple expressions for approximate values of the resonance energy has been obtained which are of importance for determination of the resonance regions where the interpolation may give erroneous values of ICC.

(v) It has been shown clearly that the occurrence of all resonances in ICC is explained by vanishing the relevant partial conversion matrix elements under the change sign. The peculiarities of behavior of the relevant matrix elements and electron wave functions at the resonance energy are considered.

(vi) Available experimental ICC ratio $\alpha_{L_{1}}^{E L} / \alpha_{L_{2}}^{E L}$ for nuclear transitions with energies near the expected position of resonances satisfactory agree with our calculations, 
which provides support for the existence of the resonances at highest $E_{\gamma}^{(\text {res })}$. For the low-energy resonances to be verified, new precise experiments are required.

\section{ACKNOWLEDGMENTS}

This work was funded by Russian Foundation for Basic Research (Project No. 09-02-00352), which is gratefully acknowledged.
[1] A. Blachowski and K. Ruebenbauer, Phys. Scr. 73, 263 (2006).

[2] Beta-Decay and Decay Heat, prepared by A. L. Nichols, International Atomic Energy Agency Report No. INDC(NDS)499, 2006.

[3] V. V. Kharitonov and V. N. Gerasimov, Phys. Solid State 40, 1462 (1998).

[4] C. Schober, V. N. Antonov, and P. Ziesche, Phys. Status Solidi B 159, 771 (1990).

[5] J. W. Petersen, G. Weyer, A. Svane, E. Holzschuh, and W. Kundig, Nucl. Instrum. Methods B 63, 179 (1992).

[6] I. M. Band and M. B. Trzhaskovskaya, At. Data Nucl. Data Tables 55, 43 (1993).

[7] Alexei M. Frolov, Radiat. Phys. Chem. 72, 541 (2005).

[8] Ch. Theisen, A. Lopez-Martens, and Ch. Bonnelle, Nucl. Instrum. Methods A 589, 230 (2008).

[9] B. R. Beck, J. A. Becker, P. Beiersdorfer, G. V. Brown, K. J. Moody, J. B. Wilhelmy, F. S. Porter, C. A. Kilbourne, and R. L. Kelley, Phys. Rev. Lett. 98, 142501 (2007).

[10] F. F. Karpeshin and M. B. Trzhaskovskaya, Phys. Rev. C 76, 054313 (2007).

[11] I. M. Band, M. B. Trzhaskovskaya, C. W. Nestor Jr., P. O. Tikkanen, and S. Raman, At. Data Nucl. Data Tables 81, 1 (2002).

[12] S. Raman, C. W. Nestor Jr., A. Ichihara, and M. B. Trzhaskovskaya, Phys. Rev. C 66, 044312 (2002).

[13] N. Nica, J. C. Hardy, V. E. Iacob, S. Raman, C. W. Nestor Jr., and M. B. Trzhaskovskaya, Phys. Rev. C 70, 054305 (2004).

[14] J. C. Hardy, N. Nica, V. E. Iacob, M. B. Trzhaskovskaya, and R. G. Helmer, Appl. Radiat. Isot. 64, 1392 (2006).

[15] N. Nica, J. C. Hardy, V. E. Iacob, W. E. Rockwell, and M. B. Trzhaskovskaya, Phys. Rev. C 75, 024308 (2007).

[16] N. Nica, J. C. Hardy, V. E. Iacob, C. Balonek, and M. B. Trzhaskovskaya, Phys. Rev. C 77, 034306 (2008).

[17] T. Kibédi, T. W. Burrows, M. B. Trzhaskovskaya, P. M. Davidson, and C. W. Nestor Jr., Nucl. Instrum. Methods A 589, 202 (2008).

[18] The BrIcc web interface is located at http://physics.anu.edu. au/nuclear/bricc.

[19] M. A. Listengarten, in Gamma Rays, edited by L. A. Sliv (Acad. Sci. USSR, Moscow/Leningrad, 1961), p. 271 (in Russian).

[20] W. B. Ewbank, Oak Ridge National Laboratory Report No. ORNL-5704, 1980.
[21] I. M. Band, M. A. Listengarten, and M. B. Trzhaskovskaya, Leningrad Nuclear Physics Institute Report No. LNPI-664, 1981.

[22] F. Rösel, H. M. Fries, K. Alder, and H. C. Pauli, At. Data Nucl. Data Tables 21, 91 (1978).

[23] O. Dragoun and M. Ry̌savý, J. Phys. G: Nucl. Part. Phys. 18, 1991 (1992).

[24] E. L. Church and J. E. Monahan, Phys. Rev. 98, 718 (1955).

[25] O. Dragoun, M. Ry̌savý, and C. Günther, Phys. Rev. C 47, 870 (1993).

[26] L. A. Sliv, Zh. Eksp. Teor. Fiz. 21, 770 (1951).

[27] J. A. Nelder and R. Mead, Comput. J. 7, 308 (1964).

[28] I. M. Band, L. A. Sliv, and M. B. Trzhaskovskaya, Nucl. Phys. A156, 170 (1970).

[29] The ENSDF database is located at http://www.nndc.bnl.gov/ ensdf/.

[30] G. A. Shevelev, A. G. Troitskaya, and V. M. Kartashov, Izv. Akad. Nauk SSSR, Ser. Fiz. 42, 211 (1978) [Bull. Acad. Sci. USSR, Phys. Ser. 42, No.1, 184 (1978)].

[31] G. G. Kennedy, S. C. Gujrathi, and S. K. Mark, Phys. Rev. C 12, 553 (1975).

[32] K. Takahashi, M. McKeown, and G. Scharff-Goldhaber, Phys. Rev. 137, B763 (1965).

[33] K. Y. Gromov, M. Honusek, C. Vylov, V. Hnatowicz, V. Zvolska, J. Zvolsky, T. A. Islamov, and A. S. Khamidov, Z. Phys. A 277, 395 (1976).

[34] J. Treherne, J. Vanhorenbeeck, and J. Valentin, Nucl. Phys. A131, 193 (1969).

[35] S. G. Malmskog and A. Backlin, Ark. Fys. 39, 411 (1969).

[36] Ch. Vieu, A. Peghaire, and J. S. Dionisio, Rev. Phys. Appl. (Paris) 8, 231 (1973).

[37] B. Rosner, J. Felsteiner, H. Lindeman, and D. Zellermayer, Nucl. Phys. A172, 643 (1971).

[38] G. Andersson and I. Bergstrom, Nucl. Phys. 3, 506 (1957).

[39] M. Kanbe, M. Fujioka, and K. Hisatake, J. Phys. Soc. Jpn. 38, 917 (1975).

[40] B. S. Dzhelepov, R. B. Ivanov, M. A. Mikhailova, and V. O. Sergeev, Izv. Akad. Nauk SSSR, Ser. Fiz. 36, 2080 (1972) [Bull. Acad. Sci. USSR, Phys. Ser. 36, 1832 (1973)].

[41] C. Briancon and R. Walen, J. Phys. (Paris) 30, 753 (1969).

[42] D. L. Spenny and A. A. Bartlett, University of Colorado Report No. COO-535-620, 1970.

[43] O. Helene and V. R. Vanin, Nucl. Instrum. Methods A 481, 626 (2002). 